\title{
Distributed estimation and control of a four-tank process
}

\author{
Awatef K Ali ${ }^{1}$ and Magdi S Mahmoud 2,* \\ ${ }^{1}$ Electronics Department, National Telecommunications Institute, Nasr City, Cairo-Egypt. \\ ${ }^{2}$ Control and Instrumentation Engineering Department, KFUPM, PO Box 5067, Dhahran 3126, Saudi Arabia.
}

Global Journal of Engineering and Technology Advances, 2021, 09(02), 133-142

Publication history: Received on 12 November 2021; revised on 25 December 2021; accepted on 27 December 2021

Article DOI: https://doi.org/10.30574/gjeta.2021.9.3.0164

\begin{abstract}
A multivariable process of four interconnected water tanks is considered for modeling and control. The objective of the current study is to design and implement a distributed control and estimation (DEC) for a multivariable four-tank process. Distributed model and inter-nodal communication structure are derived from global state-space matrices, thus combining the topology of plant flow sheet and the interaction dynamics across the plant subunits. Using experimental data, the process dynamics and disturbance effects are modeled. A typical lab-scale system was simulated and the obtained results demonstrated the potential of the DEC algorithm.
\end{abstract}

Keywords: Distributed control and estimation; Interconnected systems; Multivariable four-tank process; Four-tank system.

\section{Introduction}

A large-scale system is usually constructed from many distributed subsystems, which are interconnected with each other. Such systems are widely employed in practice, for example, multi-axis machinery, electric power systems, chemical reactors, petrochemical systems etc. [1]. Nonlinear interconnected systems are one of the most difficult to control in the category. State estimation and control algorithms have been implemented for dynamical systems represented by state-space models [2]. For highly nonlinear processes, it turns out that the linearization of high order state-space models is critical and non-trivial computational step in developing the state estimator. In addition, the centralized methods do not exploit the underlying structural features present in typical plant-wide systems. The computational burden of computing the Kalman filter renders the conventional centralized algorithm unsuitable for on-line applications to large-scale systems. The off-line computational efficiency is also an issue, albeit to a lesser degree, as a typical filter tuning approach involves computing the Kalman filter for various values of process and measurement noise covariance in search of a reasonable estimator for the application of interest. Similar results apply for linear quadratic control (LQR) control design in computing the feedback controller gain with the corresponding tuning of error penalty functions [3], [4], [7], [10].

Recent results in multi-sensor data fusion [5] employ information theoretic principles to transform the estimation and control problem to yield a fully distributed and decentralized estimation and control structure. This algorithm provides the required scalability while at the same time retain the global optimal performance that is equivalent to that of a centralized fusion system. Distributed and decentralized estimation and control (DDEC) has been successfully applied to several low-order mechanical and aeronautical systems.

\footnotetext{
* Corresponding author: Magdi S Mahmoud; E-mail:msmahmoud@kfupm.edu.sa

Control and Instrumentation Engineering Department, KFUPM, PO Box 5067, Dhahran 3126, Saudi Arabia, 


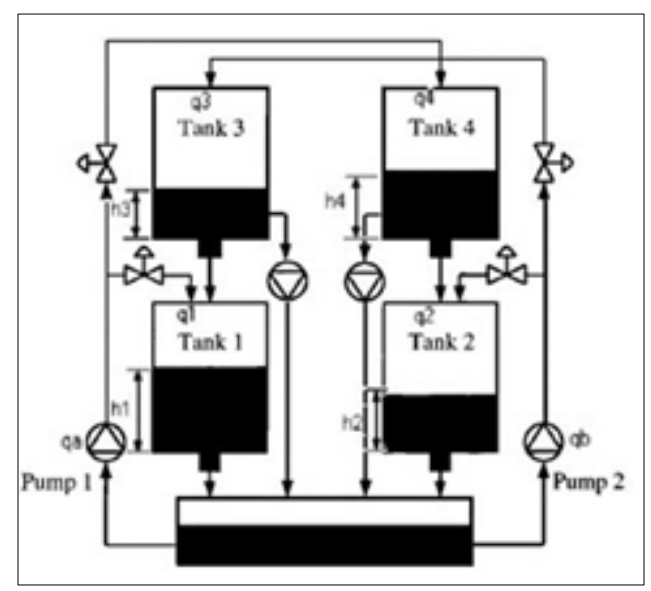

Figure 1 Schematic of the four-interconnected-tanks system

In this paper, we examine a system of four interconnected water tanks for the purpose of applied modeling and control. We then develop an improved distributed estimation and control algorithm that guarantee prescribed performance, tracking properties and provides disturbance rejection capabilities. The ensuing simulation results illustrated the efficacy of the developed method.

\section{System description}

One of the realistic process system applications is the interacting four-tank system depicted in Fig. 1, where the objective is to adjust the liquid level to prescribed value using appropriate technique.

In this system, two pumps are used to convey water from a basin into four overhead tanks. The two tanks at the upper level drain freely into the two tanks at the bottom level. The liquid levels in the bottom two tanks are measured. The piping system is such that each pump affects the liquid levels of both measured tanks. A portion of the flow from one pump is directed into one of the lower-level tanks (where the level is monitored). The rest of the flow from a single pump is directed to the overhead tank that drains into the other lower- level tank. By adjusting the bypass valves of the system, the amount of interaction between the inputs and the outputs can be varied. The process flow sheet is displayed in Fig. 1. In the present study, additional flow disturbances are introduced into the upper-level tanks. These external unmeasured disturbance flows can either drain or fill the top tanks. The process variations include uncertainties in the actuators, valve settings, and head losses in the tanks.

$$
\begin{aligned}
& \frac{\mathrm{d} h_{1}}{\mathrm{~d} t}=-\frac{a_{1}}{A_{1}} \sqrt{2 g h_{1}}+\frac{a_{3}}{A_{1}} \sqrt{2 g h_{3}}+\frac{\gamma_{1} k_{1}}{A_{1}} v_{1} \\
& \frac{\mathrm{d} h_{2}}{\mathrm{~d} t}=-\frac{a_{2}}{A_{2}} \sqrt{2 g h_{2}}+\frac{a_{4}}{A_{2}} \sqrt{2 g h_{4}}+\frac{\gamma_{2} k_{2}}{A_{2}} v_{2} \\
& \frac{\mathrm{d} h_{3}}{\mathrm{~d} t}=-\frac{a_{3}}{A_{3}} \sqrt{2 g h_{3}}+\frac{\left(1-\gamma_{2}\right) k_{2}}{A_{3}} v_{2}-\frac{k_{d_{1}} d_{1}}{A_{3}} \\
& \frac{\mathrm{d} h_{4}}{\mathrm{~d} t}=-\frac{a_{4}}{A_{4}} \sqrt{2 g h_{4}}+\frac{\left(1-\gamma_{1}\right) k_{1}}{A_{4}} v_{1}-\frac{k_{d_{2}} d_{2}}{A_{4}}
\end{aligned}
$$

Figure 2 Nonlinear model equations for the four-tank system

A nonlinear mathematical model for a similar four tanks system is detailed in [6]. The model used in the present study includes the disturbance effect of flows in and out of the upper-level tanks 3 and 4 . The differential equations representing the mass balances, based on Bernoulli's law flowing out of the tanks, in the four-tank system are given in Fig. 2 and the definition of the variables is given in Table 1. 
Table 1 Definition of variables

\begin{tabular}{|l|l|}
\hline Variable & Definition \\
\hline$h_{i}$ & the liquid level in tank i \\
\hline$a_{i}$ & the outlet cross - sectional area of tank i \\
\hline$A_{i}$ & the cross - sectional area of tank i \\
\hline$v_{j}$ & the speed setting of pump j \\
\hline$d_{1}$ & $\begin{array}{l}\text { flow disturbances from tank } 3 \\
\text { with corresponding gain } \mathrm{k}_{\mathrm{d} 1}\end{array}$ \\
\hline$d_{2}$ & $\begin{array}{l}\text { flow disturbances from tank } 4 \\
\text { with corresponding gain kd2 }\end{array}$ \\
\hline$k_{j} c_{j}$ & $\begin{array}{l}\text { gains which are the portion of the } \\
\text { flow that goes into the upper tank from pump j }\end{array}$ \\
\hline$v_{1} v_{2}$ & $\begin{array}{l}\text { The process manipulated inputs } \\
\text { (speed settings to the pumps) }\end{array}$ \\
\hline$y_{1} y_{2}$ & $\begin{array}{l}\text { The measured outputs } \\
\text { (voltages from level measurement devices) }\end{array}$ \\
\hline
\end{tabular}

It is assumed henceforth that the measured level signals are proportional to the true levels, that is, $y_{1}=k_{m 1} h_{1}$ and $y_{2}=k_{m 2} h_{2}$. The level sensors were calibrated so that $k_{m 1}=k_{m 2}=1$.

The relationship between the flows at each outlet pipe and the total flow from pump A and pump B depends on the flow parameters $\gamma_{1}$ and $\gamma_{2}$ as:

$$
q_{1}=\gamma_{1} q_{a}, q_{2}=\gamma_{2} q_{b}, q_{3}=\left(1-\gamma_{2}\right) q_{a}, q_{4}=\left(1-\gamma_{1}\right) q_{b}
$$

which are the water flows to each tank. The flow parameters $\gamma 1$ and $\gamma 2$ are such that $0 \leq \gamma 1 \leq 1,0 \leq \gamma 2 \leq 1$. The steady-state operating conditions of $v 1=50 \%$ and $2=50 \%$ are used for subsequent modeling and controller synthesis. To work with a linear description the model has to be linearized around an operating point. Defining the operating point as $h_{i}^{0}$ and the perturbation variables as $x_{i}=h_{i}-h_{i}^{0}, u_{j}=q_{j}-q_{j}^{0}$ where $j=a, b$ and $i=$ $1, \ldots, 4$. This leads to $T_{i}=\frac{A_{i}}{a_{i}} \sqrt{\frac{2 h_{i}^{0}}{g}}$.

\section{Linearized model}

\begin{tabular}{|cll|}
\hline symbol & \multicolumn{1}{c|}{ state/parameters } & \multicolumn{1}{c|}{ value } \\
\hline$h^{0}$ & nominal levels & $16.3,13.7,6.0,8.1 \mathrm{~cm}$ \\
$v^{0}$ & nominal pump settings & $50.50 \%$ \\
$a_{i}$ & area of the drain in tank i & $2.05,2.26,2.37,2.07$ \\
& & $\mathrm{~cm}^{2}$ \\
$A_{1}$ & areas of the tanks & $730 \mathrm{~cm}^{3}$ \\
$\gamma_{1}$ & ratio of flow in tank 1 to flow in tank 4 & 0.3 \\
$\gamma_{2}$ & ratio of flow in tank 2 to flow in tank 3 & 0.3 \\
$k_{j}$ & pump proportionality constants & $7.45,7.30 \mathrm{~cm}^{2} /(5 \%)$ \\
$K_{d j}$ & disturbance gains & $0.049,0.049$ \\
$T_{i}$ & time constants in the linearized model & $65.54 .1 .34 .45 .3 \mathrm{~s}$ \\
$\bar{u}, \bar{e}, \bar{r}$ & scaling factors & $25 \% .4 \mathrm{~cm} .4 \mathrm{~cm}$ \\
$g$ & Gravitation constant & $981 \mathrm{~cm} / \mathrm{s}^{2}$ \\
& & \\
\hline
\end{tabular}

Figure 3 Values of nominal parameters 
Based on the nominal values given in Fig. 3, a linearized state-space model is presented in Fig. 4 where $x=$ $\left[h_{1}, h_{2}, h_{3}, h_{4}\right], u=\left[v_{1}, v_{2}\right], d=\left[d_{1}, d_{2}\right]$, and $y=\left[h_{1}, h_{2}\right]$. The corresponding linear transfer function matrix has the form in Fig. 5. Note that individual transfer functions in each of the input - output channels of $G(s)$ do not have zeros. The right half plane zero of the system is a multivariable characteristic and imposes limitations on achievable performance.

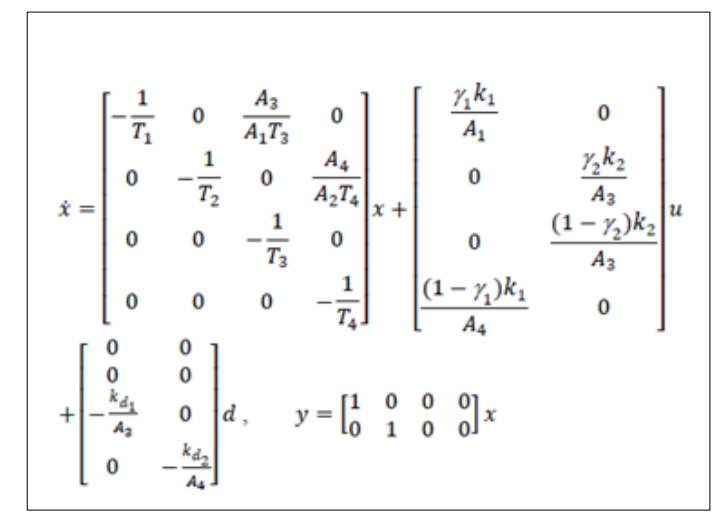

Figure 4 State space model

$$
\begin{gathered}
G(s)=\left[\begin{array}{cc}
\frac{\gamma_{1} c_{1}}{\left(T_{1} s+1\right)} & \frac{\left(1-\gamma_{2}\right) c_{2}}{\left(T_{1} s+1\right)\left(T_{3} s+1\right)} \\
\frac{\left(1-\gamma_{1}\right) c_{1}}{\left(T_{2} s+1\right)\left(T_{4} s+1\right)} & \frac{\gamma_{2} c_{2}}{\left(T_{2} s+1\right)}
\end{array}\right] \\
c_{j}=\frac{T_{j} k_{j}}{A_{j}}, \quad j=1,2
\end{gathered}
$$

Figure 5 Transfer function matrix

\section{DEC scheme}

A distributed system is defined as a data processing system in which all information is treated locally without the presence of a central processing site. Each data fusion node obtains local observations; shares appropriate information with the other nodes, assimilates the received information and computes a globally optimal estimate. A schematic diagram of a typical distributed estimation and control (DEC) network for four interconnected tank system is shown in Fig. 6.

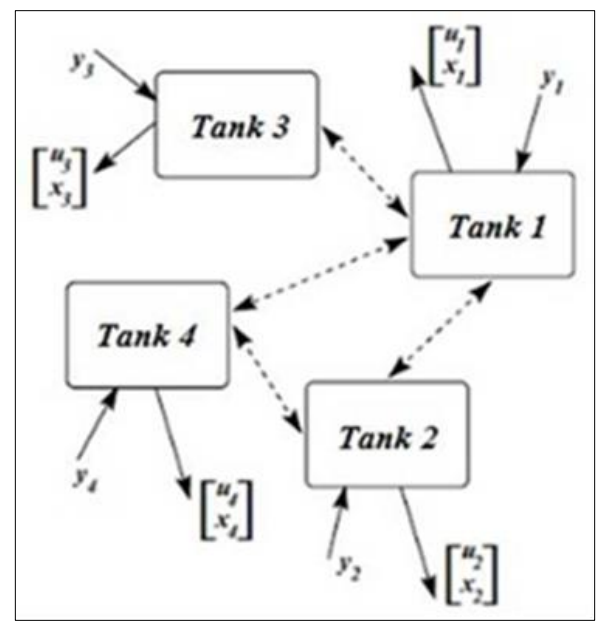

Figure 6 A typical distributed control and estimation structure 


\begin{tabular}{|c|l|}
\hline Variable & Definition \\
\hline$x(k)$ & $x(k) \in R^{n}$, the n-dimensional state of interest at time $k$ \\
\hline$\Phi$ & $\begin{array}{l}\Phi \in R^{n} \rightarrow R^{n} \text { is the state transition matrix from time } k \\
\text { to } k-1 .\end{array}$ \\
\hline$B$ & $\mathrm{~B} \epsilon R^{n} \rightarrow R^{n}$, the input control matrix \\
\hline$u(k)$ & $u(k) \epsilon R^{n} \rightarrow R^{n}$, the input control vector \\
\hline$y(k)$ & $y(k) \in R^{n}$, the vector of measurements at time $k$ \\
\hline$C$ & $H \in R^{n} \rightarrow R^{n}$, is the observation matrix \\
\hline$v(k)$ & $\begin{array}{l}v(k) \sim N(0, R), \text { the associated measurements noise, } \\
\text { uncorrelated, zero mean, white sequences with } \\
\text { corresponding covariance matric } \mathrm{R} .\end{array}$ \\
\hline$w(k)$ & $\begin{array}{l}w(k) \sim N(0, Q), \text { the associated process noise, } \\
\text { uncorrelated, zero mean, white sequences with } \\
\text { corresponding covariance matric Q. }\end{array}$ \\
\hline
\end{tabular}

Figure 7 Definition of variables in model (2)

A linear discrete-time system with $n_{u}$ inputs and $n_{y}$ outputs of the following state-space form is considered:

$$
x(k)=\Phi x(k-1)+B u(k-1)+w(k-1)
$$

$y(k)=C x(k)+v(k)$

where the definition of the variables involved is listed in Fig. 7.

The proposed DEC scheme can be divided into three main phases:

- Model Decomposition

- $\quad$ Distributed Estimation

- Prediction

- Estimation

- $\quad$ Distributed Control

\subsection{Model decomposition}

The preliminary step in the design of the DDEC network is the distribution of the state prediction and observation equations across a network of $N$ number of processing nodes. The local state vector at node i; $x_{i}(k)$ is related to the global state vector $x(k)$ by $x_{i}(k)=T_{i} x(k)$; where $T_{i}$ is a linear nodal transformation matrix. Through the inter-nodal transformations $T_{i}$ only the locally relevant states for each node are chosen. Relevance of states is defined by the global state transition matrix $\Phi$; which incorporates the effects of states on each other as the system evolves in time. Modelspecific inter-nodal communication ensures that only appropriate nodes communicate relevant information. The local state transition and observations at node i are given by:

$$
\begin{aligned}
& x_{i}(k)=\Phi_{i} x_{i}(k-1)+B_{i} u_{i}(k-1)+w_{i}(k-1) \\
& y_{i}(k)=C_{i} x_{i}(k)+v_{i}(k)
\end{aligned}
$$

where $u_{i}(k)$ are the inputs affecting the local states and $\Phi_{i}$ is related to the global state transition matrix $\Phi$ with the following relation $\Phi_{i}=T_{i} \Phi T_{i}^{*}, T_{i}^{*}$ is a generalized inverse of $T_{i}$. The observation matrix $C_{i}$ is periodic with time period of $N_{\mathrm{m}}$ samples and is non-zero only at every $T N_{m}^{t h}$ sample instant. There are points need to be considered when we want to partition the process into estimation nodes:

- $\quad$ Similarity of the estimation network to the actual plant.

- The computational load at each node.

- The communication overhead.

- The available computational resources.

- Avoid excessive partitioning otherwise communication load will increase and the computational burden on will decrease on each node.

The procedure for constructing a distributed estimation network can be done as follows: 
- A Identify the "states of interest" which are the states of the process unit and assign one estimation node to each of the identified units.

- B Obtain the discretized model from the continuous time description. The sample time DT for discretization is critical. For small DT; the hierarchy in the discretized model is same as that in the continuous description. However, low DT results in high communication rates between nodes.

- C Identify the "overlapping" states at each node based on the discrete-time global state transition matrix, if available. Otherwise, the process flow sheet and the system matrices of individual units could be used. This step will determine the connectivity of the network and the number of states in the model at each estimation node. The "relevant states" that constitute the local model are the"states of interest" augmented by the "overlapping states".

- D If necessary, combine and partition nodes further to balance computational load and communication overhead across all the nodes in the network.

- E If the communication is excessive due to large number of "overlapping" states, repeat the decomposition process with a lower discretization sample time, to be explored on a trial-and error basis.

- $\quad$ F To the extent possible, limit the number of nodes based on the available computational resources.

The distributed estimation and control network thus designed is not necessarily fully connected. Various ring, tree or loop connected topologies can result based on the inter-nodal transformations defined by the model.

\subsection{Distributed estimation}

In this phase, we use the distributed and decentralized Kalman filter (DDKF) which is done in two stages:

\section{Prediction}

At each node the following prediction steps are performed locally prior to any communication with the other nodes.

$$
\begin{aligned}
& \hat{x}_{i}(k \mid k-1)=\Phi_{i} \mid \hat{x}_{i}(k-1 \mid k-1)+B_{i} u_{i}(k-1) \\
& \hat{P}_{i}(k \mid k-1)=\Phi_{i} P_{i}(k-1 \mid k-1) \Phi_{i}^{T}+Q_{i}
\end{aligned}
$$

Estimation

The estimation step consists of three stages:

- Local estimation,

- Inter-nodal communication

- Assimilation to produce global estimate.

Local covariance and state estimates are computed based on local measurements as:

$$
\begin{array}{r}
P_{j}\left(k \mid y_{j}(k)\right)=\left[C_{j}^{T} R_{j}^{*} C_{j}\right]^{*} \Phi_{i} \hat{x}_{i}(k \mid k-1)+B_{i} u_{i}(k-1) \\
\hat{x}_{j}\left(k \mid y_{j}(k)\right)=P_{j}\left(k \mid y_{j}(k)\right)\left[C_{j}^{T} R_{j}^{*} C_{j}\right] \times\left[\epsilon_{j}(k)+C_{j}\left(N_{m}\right) x_{j}(k \mid k-1)\right]
\end{array}
$$

where $\epsilon_{j}(k)$ is the innovation at time $k$ defined by $\epsilon_{j}(k)=y_{i}(k)-C_{j}\left(N_{m}\right) x_{j}(k \mid k-1)$, for $k=0, N_{m}, 2 N_{m}, \ldots$ and $\epsilon_{j}(k)=$ 0 , otherwise. The relevant subset of local estimates of state and prediction error covariance's are communicated to relevant nodes and the information at each node is transformed into local state subspace.

The transformed state and covariance estimates are given by

$$
\begin{aligned}
& \left.\hat{x}_{i}(k \mid k)=P_{i}(k \mid k)\left[P^{(}-1\right)_{i}(k \mid k-1) \hat{x}_{i}(k-1 \mid k-1)\right]+\sum_{j=1}^{N} P_{i}^{*}\left(k \mid y_{j}(k)\right) \hat{x}_{i}\left(k \mid y_{j}(k)\right) \\
& \left.P_{i}(k \mid k)=\left[P^{(}-1\right)_{i}(k \mid k-1)+\sum_{j=1}^{N} P_{i}^{*}\left(k \mid y_{j}(k)\right)\right]^{*}
\end{aligned}
$$

\subsection{Distributed control}


A nodal control law obtained as a cost minimizing control function is given by

$$
u_{i}(k)=K_{c i}\left[x_{r i}(k)-\hat{x}_{i}(k \mid k)\right]
$$

where $x_{r i}(k)$ is the local state reference, and $\hat{x}_{i}(k \mid k)$ is the local optimal state estimate, and $K_{c i}$ is the optimal control gain computed from the solution to a distributed backward Riccati recursion given as

$$
\begin{gathered}
K_{c i}(k)=\left[\Gamma_{u i}+B_{i}^{T}(k) \Psi_{i}(k) B_{i}(k)\right]^{-1} \times B_{i}^{T}(k) P s i_{i}(k) \Phi_{i} \\
B_{i}(k)=T_{i}\left[B K_{c}(k)\right] T_{i}^{*} K_{c i}^{*}(k) \\
K_{c}(k)=B^{*} T_{i}^{*}\left[B_{i}(k) K_{c i}(k)\right] T_{i} \\
\Psi_{i}(k)=\Gamma_{x i}\left[\Phi_{i}^{T} \Psi_{i}(k+1)\right]\left[\Phi_{i}-B_{i}(k) K_{c i}(k)\right]
\end{gathered}
$$

where $\Gamma_{\text {ui }}$ and $\Gamma_{\text {xi }}$ are local state and control cost weighting matrices, respectively.

Schematic representation of the prediction, communication, and assimilation stages in an individual node in the distributed estimation and control network is portrayed in Fig. 8.

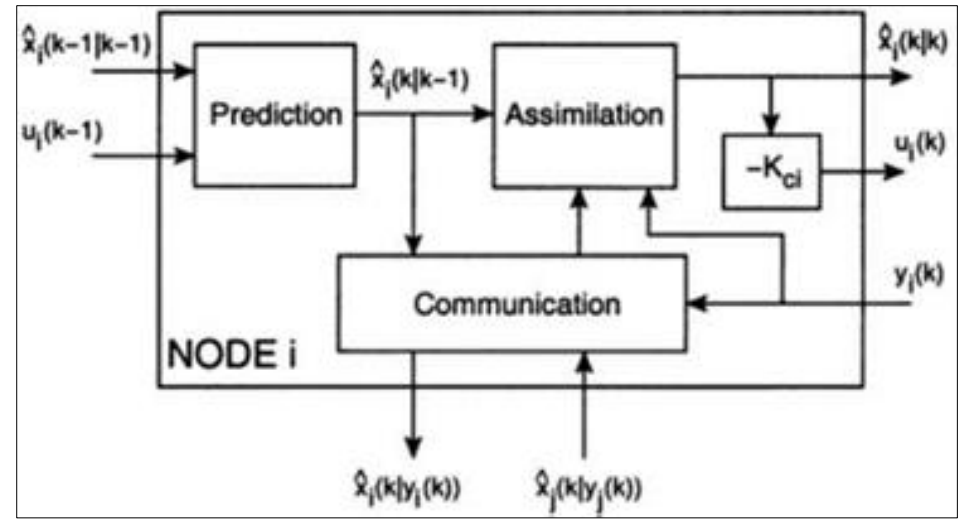

Figure 8 Prediction and estimation structure

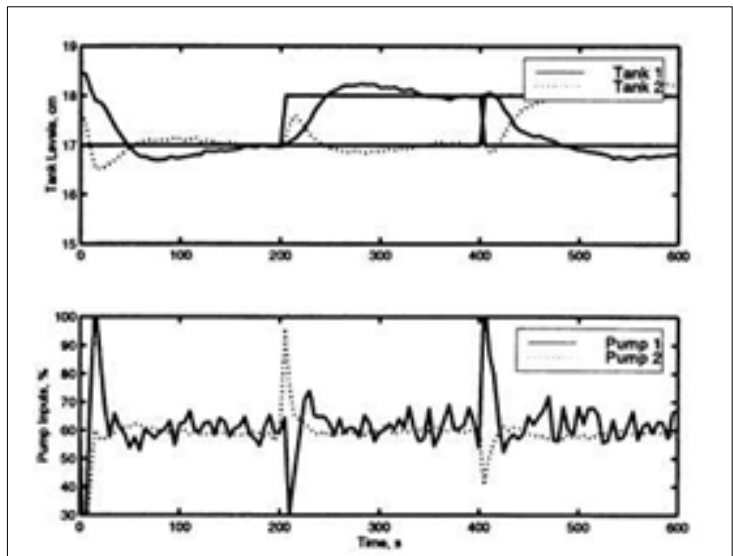

Figure 9 Closed-loop reference tracking using DEC algorithm

\section{Simulation results}

In computer experimentation, MATLAB R2008b was used to implement the control algorithms on DELL Latitude 6400, $2.4 \mathrm{GHz}$ personal laptop with $2 \mathrm{~GB}$ of memory with operating system Windows Vista Business. The sampling time for the interconnected tank system is $0.1 \mathrm{~s}$ and the time constant is about $30 \mathrm{~s}$. Initially, Fig. 9 shows closed-loop performance of the DEC algorithm for reference changes. 
Then two sets of computer simulation experiments were carried out:

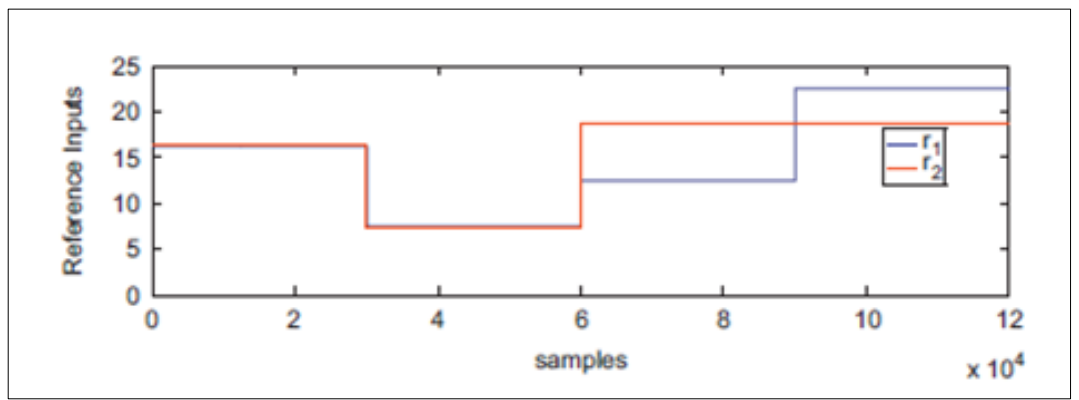

Figure 10 Set points

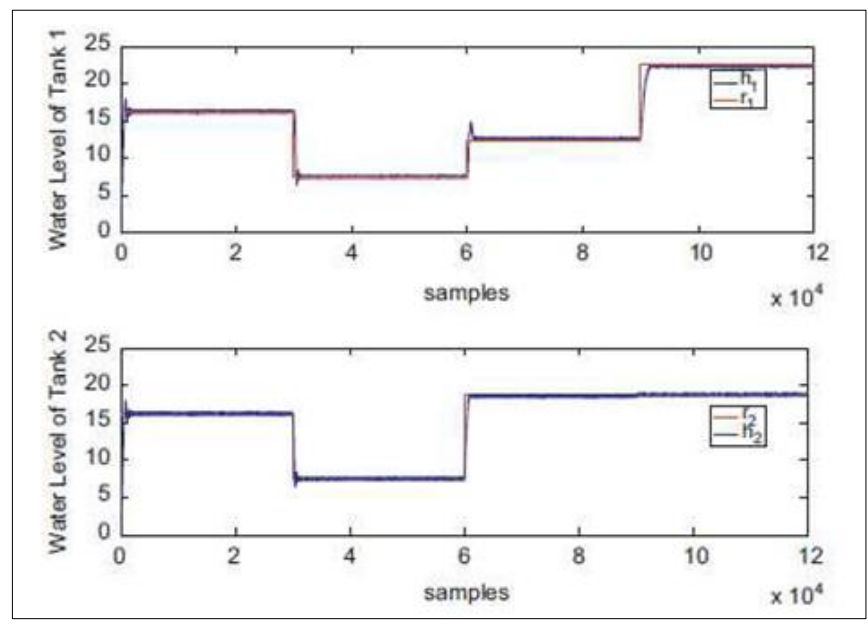

Figure 11 Water level variations using DEC algorithm

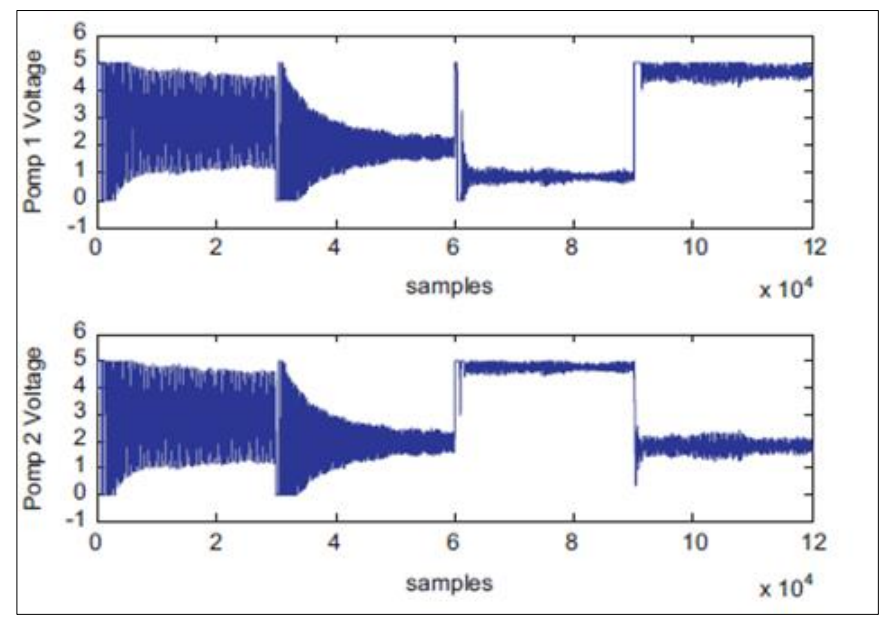

Figure 12 Pump voltage variations using DEC algorithm

\subsection{Simulation run 1}

Given a particular weighting matrices $P_{i}=I, Q_{i}=0$, the set point signals are given in Fig. 10 and the corresponding water level and pump voltage variations are plotted in Figs. 11 and 12, respectively.

\subsection{Simulation run 2}

Given a different weighting matrices $\mathrm{Pi}=\mathrm{I}, \mathrm{Qi}=10 \mathrm{I}$, the set point signals are given in Fig. 10 and the corresponding water level and pump voltage variations are plotted in Figs. 13 and 14, respectively. 


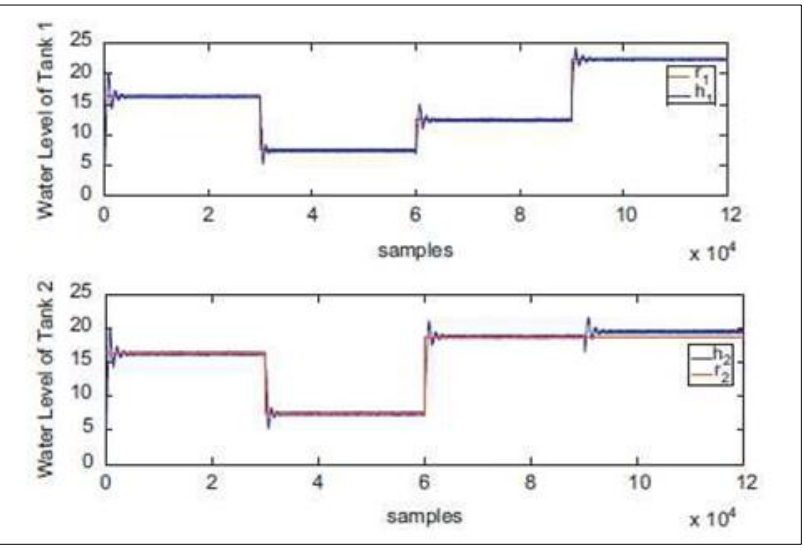

Figure 13 Water level variations using DEC algorithm

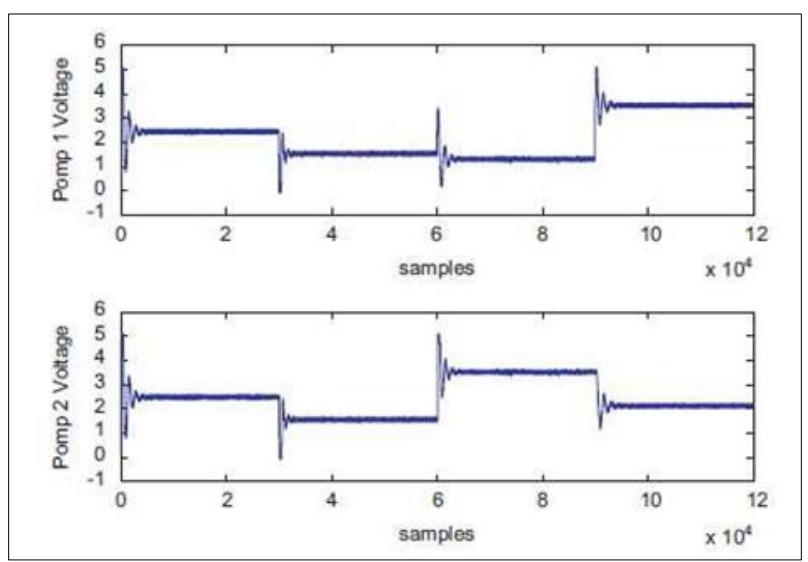

Figure 14 Pump voltage variations using DEC algorithm

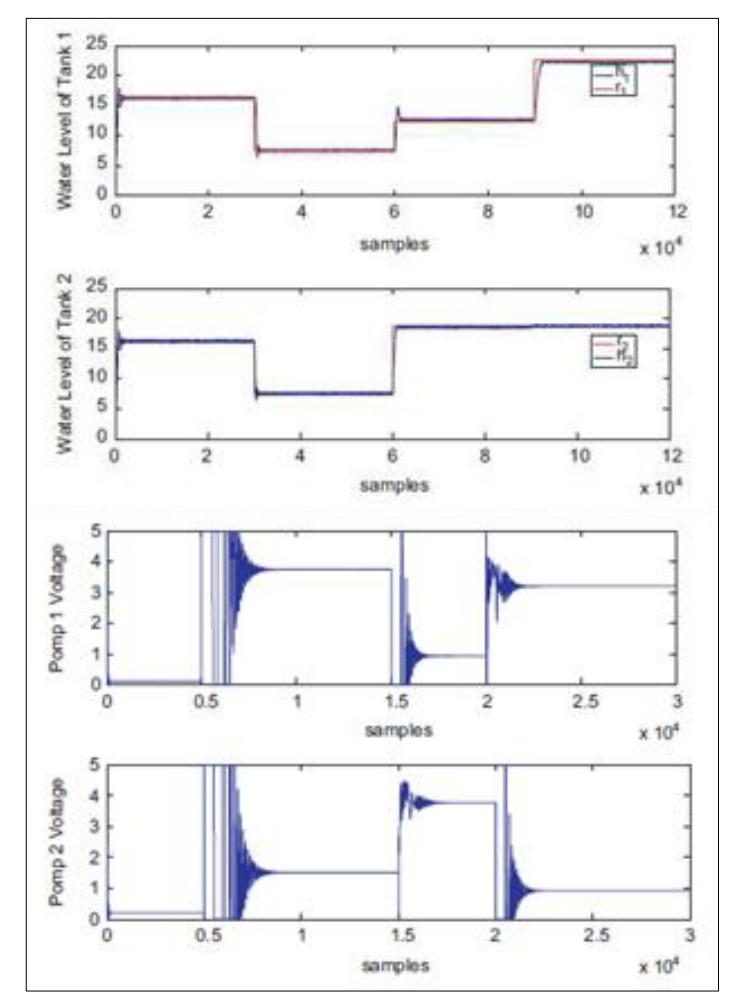

Figure 15 Disturbance rejection results 
From the ensuing results, it is observed that the maximum and minimum levels of control signal are 0 and $5 \mathrm{~V}$, respectively, and the maximum levels of tanks are $25 \mathrm{~cm}$. The controller designed in the second simulation run illustrated that the variations of the control signals are successfully limited. This second controller brings tracking error during the last step (from samples $9--104$ to $12--104$ ). This shows the inability of the second controller to overcome the interaction among loops.

The obtained results illuminated very good performance, which enhances the importance of incorporating an estimator that faithfully reproduce the system states. In addition, the developed controllers designed by the DEC algorithm significantly track the reference input.

Finally, the developed method has been evaluated for disturbance rejection problem. Fig. 15 shows the results for the DEC methodology in a disturbance rejection problem. In this test, following [8], [9] the input references have been applied at sample 5000 , then in sample 15, 000 the disturbance input on h1 with amplitude $5 \mathrm{~cm}$ has been applied. The same disturbance has been applied to the second output at sample 20, 000. As depicted in Fig. 15 the controller has demonstrated good properties in the closed-loop experiments, exhibiting stable and feasible trajectories in spite of the disturbances.

\section{Conclusion}

In this paper, we have shown one application of distributed estimation and control (DEC) methodology in designing an effective level control system. The methodology is implemented on an interconnected tank system. The simulation results have emphasized the potential of the developed DEC for process control industry.

\section{Compliance with ethical standards}

\section{Acknowledgments}

The authors would like to thank the deanship for scientific research (DSR) at KFUPM for research support through distinguished professorship award project no. IN 191006.

\section{Disclosure of conflict of interest}

The authors of the paper admit that there is conflict of interest.

\section{References}

[1] MS Mahmoud, MA Darwish, MF Hassan. Large-Scale Control Systems: Theories and Techniques, New York: Marcel Dekker. 1985.

[2] MS Mahmoud, Y Xia. Applied Control Systems Design: State- Space Methods, Springer-Verlag, UK. 2012.

[3] Y Alipouri, J Poshtan. Optimal controller design using discrete linear model for a four tank benchmark process, ISA Transactions. 2013; 52: 644-651.

[4] EP Gatzke, ES Meadows, C Wang, FJ Doyle III. "Model based control of a four-tank system", Computers and Chemical Engineering. 2000; 24: 1503-1509.

[5] MS Mahmoud, Y Xia. Networked Filtering and Fusion in Wireless Sensor Networks, CRC Press (Taylor and Francis Group), New York. December 2014.

[6] KH Johansson, JLR Nunes. "A multivariable laboratory process with an adjustable zero", Proc. American Control Conference, Philadelphia, PA. 1998; 2045-2049.

[7] KH Johansson. The quadruple-tank process: A multivariable labo- ratory process with an adjustable zero, IEEE Trans. Control Systems Technology. 2000; 8(3).

[8] R Ostermark. Genetic hybrid tuning of VARMAX and state space algorithms, Soft Computing. 2010; 14: 91-99.

[9] JM. Maciejowski, Multivariable Feedback Design, Addison-Wesley Publishing Company. 1989.

[10] I Alvarado, D Limon, D Munoz, DL Pena, MJ Maestre, MA Ridao. “A comparative analysis of distributed MPC techniques applied to the HD-MPC four-tank benchmark”, Journal of Process Control, vol. 21, pp. 800-815, 2011. 